\title{
National survey of percutaneous coronary intervention during the COVID-19 pandemic in Japan: second report of the Japanese Association of Cardiovascular Intervention and Therapeutics
}

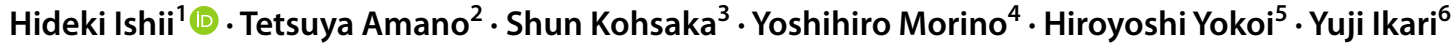

Received: 7 April 2021 / Accepted: 9 April 2021 / Published online: 17 April 2021

(c) Japanese Association of Cardiovascular Intervention and Therapeutics 2021

\begin{abstract}
Healthcare systems worldwide have been overburdened by the coronavirus disease 2019 (COVID-19) outbreak. Accordingly, hospitals have had to implement strategies to profoundly reorganize activities, which have affected procedures such as primary percutaneous coronary interventions (PCIs). This study aimed to describe changes in PCI practices during the health emergency at the national level. The Japanese Association of Cardiovascular Intervention and Therapeutics performed provided serial surveys of institutions throughout Japan during the pandemic. The data obtained on December, 2020 and February 2021 (during the 2nd wave of pandemic) were compared with the data obtained on August 2020 (1st wave). Primary PCI for STEMI was performed as usual in 99.1\%,98.7\%, and 97.5\% of institutions in mid-August, mid-December, 2020 and mid-February, 2021, respectively. The COVID-19 screening tests rates in patients were significantly higher during the third wave than during the second wave $(54.0 \%$ in mid-August, 2020 and $64.6 \%$ in mid-February, 2021, $P=0.002)$. In addition, hospitals reported that personal protective equipment was more available over time (66.4\% in mid-August, 2020 and $83.8 \%$ in mid-February, 2021, $P<0.001)$. In conclusion, most institutions surveyed in Japan continued to perform primary PCI as usual for STEMI patients during the second and third waves of the COVID-19 pandemic. In addition, the COVID-19 screening tests were more frequently performed over time.
\end{abstract}

Keywords COVID-19 $\cdot$ Percutaneous coronary intervention $\cdot$ ST elevation myocardial infarction $\cdot$ Survey $\cdot$ Japan

\section{Introduction}

Coronavirus disease 2019 (COVID-19), caused by the severe acute respiratory syndrome coronavirus 2 (SARS-CoV-2), has been an unprecedented challenge for health care systems. Initial reports from Wuhan, China, suggested that the SARS-CoV-2 was highly transmissible, posing significant challenges to prevention, containment, and treatment. Hospital systems worldwide, therefore, had to brace for the impact of COVID-19 [1, 2].

In Japan, the first wave of COVID-19 infection initially peaked in April 2020, and significantly decreased by mid-late May [3]. However, the situation worsened again in late June (the beginning of the second wave) and decreased in late August, 2020. Unfortunately, the number of COVID-19 patients began to rise again in November 2020, and this has been termed the third wave of COVID19. On January 7, 2021, Prime Minister Yoshihide Suga 
declared a state of emergency for Tokyo Metropolis, Kanagawa, Saitama, and Chiba prefectures, and expanded the state of emergency to Tochigi, Osaka, Hyogo, Kyoto, Aichi, Gifu and Fukuoka prefectures on January 13 [4]. In some regions of Japan, medical care systems were on the verge of collapsing owing to the increasing number of COVID-19 patients [5]. Therefore, collecting data on the performance of direct PCIs and care of ST elevation myocardial infarction (STEMI) patients is warranted across the country.

The present study aimed to describe any changes in direct PCI practices in STEMI patients during this health crisis at the national level. During the first wave of the pandemic, three serial surveys were distributed (in mid-April, lateApril and mid-May 2020) by the Japanese Association of Cardiovascular Intervention and Therapeutics (CVIT) on decision-making for elective and direct PCIs from institutions throughout Japan. Similar additional serial surveys were distributed during the second and third waves of the COVID-19 pandemic.

\section{Methods}

\section{Survey}

After CVIT surveyed institutions throughout Japan during the first wave of the COVID-19 pandemic [6], serial surveys were distributed during the second and third waves in mid-August and mid-December 2020 and mid-February 2021). The surveys were sent to a total of 680 representative PCI centers with an annual PCI case volumes of at least 50 procedures from all 47 prefectures in Japan and included the following three types of queries regarding direct PCI in STEMI patients:

1. Preferences regarding performing primary PCI in STEMI patients.

2. Use of screening tests such as polymerase chain reaction (PCR) and chest computed tomography (CT) to detect COVID-19.

3. Availability of protective personal equipment (PPE) such as particulate respirator type N95 (N95 mask) and disposable non-woven gowns.

Only in the mid-February 2021 survey did we additionally include a question regarding whether the general emergency medical service at each institution was affected by the COVID-19 pandemic. The status in mid-December 2020 was simultaneously collected as part of the survey distributed in mid-February 2021. Definition of STEMI has been described in detail elsewhere [7].

\section{Statistical analysis}

SPSS v22 was used for all the analyses. Categorical variables were compared using the Pearson's Chi-square test. For questions regarding preferences in performing primary PCI and the application of screening tests, the serial surveys conducted during the first wave of the pandemic (in midApril, late-April and mid-May 2020) [6] were compared to the surveys conducted during the second and third waves. A $P$ value $<0.05$ was considered statistically significant.

\section{Results}

\section{Primary PCI for STEMI patients}

A total of 426 and 395 institutions responded in midAugust 2020 and mid-February 2021, respectively (Table 1). Among, 395 institutions that responded in midFebruary 2021, 237 (60.0\%) reported that they were overwhelmed by COVID-19 patients (e.g. there were limitations in emergency services). Regardless, primary PCI for

Table 1 Answers to the questions

\begin{tabular}{|c|c|c|c|}
\hline & $\begin{array}{l}\text { Mid-Aug, } \\
2020 \\
(n=426)\end{array}$ & $\begin{array}{l}\text { Mid-Dec, } \\
2020 \\
(n=395)\end{array}$ & $\begin{array}{l}\text { Mid-Feb, } \\
2021 \\
(n=395)\end{array}$ \\
\hline \multicolumn{4}{|l|}{ Number } \\
\hline \multicolumn{4}{|l|}{$\mathrm{PCI}$ in high risk $\mathrm{ACS}(\%)$} \\
\hline As usual & 99 & 99 & 97 \\
\hline Any limitation & 1 & 1 & 3 \\
\hline \multicolumn{4}{|c|}{ Screening for COVID-19 in STEMI (\%) } \\
\hline $\begin{array}{l}\text { Physical examination } \\
\text { only }\end{array}$ & 46 & 41 & 35 \\
\hline $\begin{array}{l}\text { PCR, chest CT, and } \\
\text { others }\end{array}$ & 54 & 59 & 65 \\
\hline PCR for COVID-19 & 18 & 35 & 48 \\
\hline Chest CT & 39 & 34 & 36 \\
\hline $\begin{array}{l}\text { Anti-gen for COVID- } \\
19\end{array}$ & 13 & 47 & 49 \\
\hline $\begin{array}{l}\text { Anti-body for COVID- } \\
19\end{array}$ & 0 & 2 & 2 \\
\hline \multicolumn{4}{|l|}{ Fill rate of PPE } \\
\hline Fully filled & 66 & N/A & 84 \\
\hline Filled but limited stock & 15 & N/A & 12 \\
\hline Not filled & 19 & N/A & 4 \\
\hline N 95 & 8 & N/A & 2 \\
\hline Gown & 11 & N/A & 2 \\
\hline
\end{tabular}

$C T$ computed tomography, $N 95$ particulate respirator type N95, PCI percutaneous coronary intervention, $P C R$ polymerase chain reaction, $P P E$ personal protective equipment, STEMI ST elevation myocardial infarction 
STEMI patients was performed as usual in $99.1 \%, 98.7 \%$, and $97.5 \%$ of institutions in mid-August 2020 mid-December 2020 and mid-February 2021, respectively (Table 1).

Serial data for all three waves of the pandemic are shown in Fig. 1. In mid-August 2020, the limitations for direct PCI was the lowest $(0.9 \%)$. There was a statistically significant difference $(P=0.002)$ over time. However, there was no significant difference between midAugust and mid-December $2020(P=0.65)$ or between
mid-August 2020 and mid-February $2021(P=0.078)$ regarding direct PCI performance rates (Fig. 1).

\section{The COVID-19 screening test}

The COVID-19 screening test rate in STEMI patients increased over time $(P<0.0001)$ (Table 1 ; Fig. 2). In midAugust 2020, the rate was $54.0 \%$, and it increased to $64.6 \%$ in mid-February $2021(P=0.002)$. PPE resources were fully available in only $66.4 \%$ of institutions in mid-August 2020 ,
Fig. 1 Performance rates of primary percutaneous coronary intervention in patients with ST elevation acute myocardial infarction

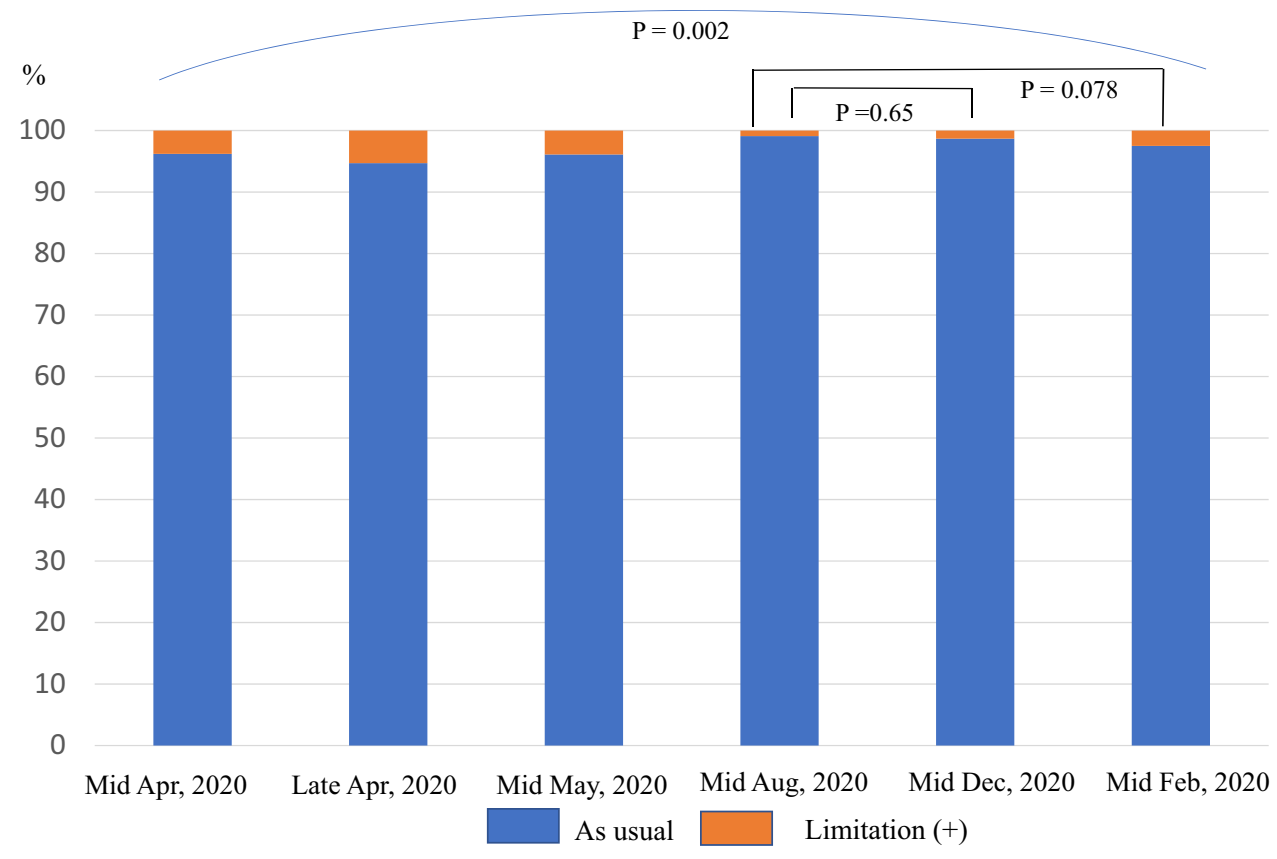

Fig. 2 Proportion of screening tests to detect COVID-19

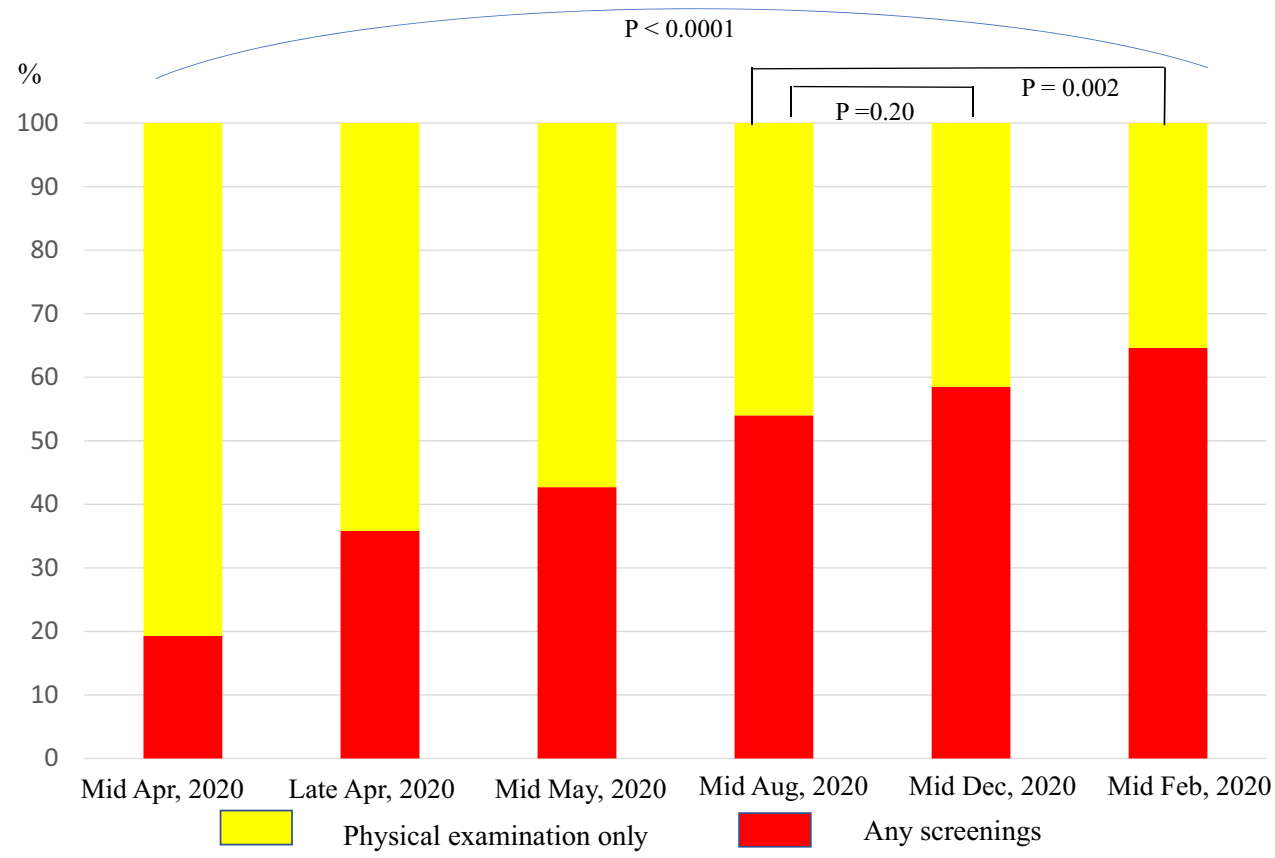


however, the percentages increased to $83.8 \%$ by mid-February $2021(P<0.001)$.

\section{Discussion}

The USA, Europe, Russia, India and other countries have been experiencing a high percentage of COVID-19 cases relative to their populations. Although the number in Japan is also increasing, as of March 19, 2021, the incidence of COVID-19 was only 359 per 100,000 people in Japan, making Japan 120th in the world for the number of infections per 100,000 [1, 8]. However, the number available hospital beds for COVID-19 patients has been limited in Japan [5] Therefore, the number of therapeutic procedures performed could likely have been affected during the second and third waves of the pandemic. After November 2020, the occupancy rates for COVID-19-designated hospital beds had markedly increasing, and emergency medical systems were nearly collapsing in some regions in Japan. However, our results showed that the most institutions continued to perform primary PCI as usual for STEMI patients during the second and third waves of the COVID-19 pandemic. Doctors and medical staff had to grapple with the great surge in infections.

Our surveys showed that COVID-19 screening tests were more frequently performed over time, especially the PCR tests. During the first wave of the pandemic, PCR tests for COVID-19 were only available in a limited number of institutions and the supply was low, presenting a particular challenge for testing and detecting COVID-19 patients [9]. However, the number of PCR tests available increased during the second and third waves of the pandemic. Although it might seem difficult to keep time fast when screening tests were performed, shortening time to confirm or rule-out COVID19 is simultaneously important in patients with STEMI. At the point, determining the ideal trade-off is essential.

The proportion of hospital admissions was lower, and the rate of in-hospital mortality was higher for acute coronary syndrome (ACS) patients in Western countries, during the COVID-19 pandemic compared to previous periods. In addition, direct PCI for STEMI was reported to have decreased by nearly $25-40 \%$ [10-12]. To date, only a few studies have assessed the number of new cases of ACS and PCI procedures in Japan. A study from Tokai region in Japan has suggested that during the first wave, a decline of $11.5 \%$ was observed in the incidence of ACS, compared to the equivalent period in 2019 and that in-hospital parameters of ACS cases, including door-to-balloon (D2B) time, were comparable between the periods [13]. In contrast, the rate of delayed admission after symptom onset in ACS patients was found to have significantly increased in Shimane and Osaka prefectures during the pandemic compared to the control period $[14,15]$. It is possible that more patients may have been denied access to the hospital since the onset of the pandemic. From this point of view, a strategy to reduce the prehospital delay in ACS cases is warranted in the critical circumstance.

In Japan, the mortality rate of acute myocardial infarction (AMI) patients, including both in-hospital and outof-hospital deaths, was reported to be $31.3 \%$ in 2019 and $37.1 \%$ in 2015 [16]. However, in the critical situations during the COVID-19 pandemic, the prehospital delay and delayed D2B time owing to the need for screening tests or wearing PPE may affect clinical prognosis in STEMI patients. Until now, there have been no current data on inhospital mortality, or prognosis in STEMI patients based on large scale or national registry. J-ROAD, J-PCI and its follow-up registry may soon address the issues described above. On the other hand, it is noteworthy that in Japan, the number of deaths in 2020 decreased to approximately 138,5000 , which means a reduction of approximately 9400 deaths compared to 2019 [17]. We should evaluate how the performance of PCI affected the decline in mortality in Japan.

Needless to say, shortening the duration of D2B time is important to improve prognosis of AMI patients [18]. Apart from the point, the Japanese health insurance charges higher medical fees for direct PCI with a D2B time $\leq 90$ min compared to that with a D2B time $>90 \mathrm{~min}$. Therefore, hospitals may be concerned about these issues because of delayed D2B time in some cases. Further investigations are also needed for the point.

Limitations should be discussed. First, the surveys were completed voluntarily, and some hospitals did not complete the survey. Therefore, the results of the study cannot be generalized to all regions of Japan. Second, the circumstance of each medical system may have differed depending on the severity of the COVID-19 outbreak or the prevalence of COVID-19 in each area. Third, the participating hospitals covered all 47 prefectures within Japan, however, the roles and characteristics of each hospital may have differed even within one region.

\section{Conclusion}

Japanese hospitals tended to perform primary PCI for STEMI patients as usual during the second and third waves of the COVID-19 pandemic. An increase in COVID-19 screening tests, particularly PCR, was more frequently observed over time. In addition, the percentage of hospitals where PPE resources were fully available gradually increased, however, approximately $15 \%$ of institutions continued to report a lack of PPE as of mid-February, 2021. 
Acknowledgements We would like to thank all the participating hospitals for their efforts on the survey, Hiroshi Takahashi, BSc for excellent assistance with the manuscript, and CVIT staffs for providing administrative support.

Funding This work was supported by the Japanese Association of Cardiovascular Intervention and Therapeutics (CVIT; Japan) and the grants-in-aid of the 24th General Assembly of the Japanese Association of Medical Sciences.

\section{Declarations}

Conflict of interest Hideki Ishii received lecture fees from Astellas Pharma, AstraZeneca, Bayer Pharmaceutical Co.Ltd., Chugai Pharma Inc., Daiichi-Sankyo, and MSD K. K. Tetsuya Amano received lecture fees from Astellas Pharma, AstraZeneca, Bayer, Daiichi Sankyo, and Bristol-Myers Squibb. Shun Kohsaka received investigator-initiated grant funding from Bayer and Daiichi Sankyo and received lecture fees from Bristol-Myers Squibb. Yoshihiro Morino received research grants and lecture fees from Boston Scientific Corporation, Terumo, Baier, Japan lifeline, and Daiichi-Sankyo, and received lecture fees from Kowa pharmaceutical and Amgen. Hiroyoshi Yokoi received lecture fees from Abbott Vascular Japan Co., Ltd., Amgen Astellas BioPharma, Astellas Pharma, Abbott Vascular Japan Co., Ltd., Bayer Pharmaceutical Co.Ltd., Boston Scientific Corporation, Daiichi Sankyo, HeartFlow Japan G.K., Otsuka Pharmaceutical Co., Ltd., Sanofi K.K., Takeda Pharmaceutical Company, and Terumo Co., Ltd. Yuji Ikari received research grant from Boston Scientific and Bayer.

IRB information This study was granted an exemption from requiring ethics approval by CVIT committee.

\section{References}

1. World Health Organization. https://www.who.int/emergencies/ diseases/novel-coronavirus-2019/situation-reports/. Accessed 20 Mar 2021.

2. Mizuno A, Matsumoto C, Yoneoka D, Kishi T, Ishida M, Sanada $\mathrm{S}$, et al. Cardiology department practices in the first wave of the coronavirus disease pandemic - a nationwide survey in Japan by the Japanese Circulation Society. Circ Rep. 2021;3:137-41.

3. Japanese Ministry of Health, Labour and Welfare. https://www. mhlw.go.jp/stf/seisakunitsuite/bunya/0000164708_00001.html. Accessed 20 Mar 2021.

4. Japanese Cabinet Secretarist https://corona.go.jp/en/. Accessed 20 Mar 2021.

5. Japanese Ministry of Health, Labour and Welfare. https://www. mhlw.go.jp/stf/seisakunitsuite/newpage_00035.html. Accessed 20 Mar 2021.

6. Ishii H, Amano T, Yamaji K, Kohsaka S, Yokoi H, Ikari Y. Implementation of percutaneous coronary intervention during the COVID-19 pandemic in Japan - nationwide survey report of the
Japanese Association of Cardiovascular Intervention and Therapeutics for cardiovascular disease. Circ J. 2020;84:2185-9.

7. Sawano M, Yamaji K, Kohsaka S, Inohara T, Numasawa Y, Ando $\mathrm{H}$, et al. Contemporary use and trends in percutaneous coronary intervention in Japan: an outline of the J-PCI registry. Cardiovasc Interv Ther. 2020;35:218-26.

8. COVID-19 Dashboard by the Center for Systems Science and Engineering (CSSE) at Johns Hopkins University (JHU). https:// gisanddata.maps.arcgis.com/apps/opsdashboard/index.html\#/ bda7594740fd40299423467b48e9ecf6. Accessed 20 Mar 2021.

9. Our World in Data. https://ourworldindata.org/covid-testi ng. Accessed 20 Mar 2021.

10. De Rosa S, Spaccarotella C, Basso C, Calabrò MP, Curcio A, Filardi PP, et al. Reduction of hospitalizations for myocardial infarction in Italy in the COVID-19 era. Eur Heart J. 2020;41:2083-8.

11. Mafham MM, Spata E, Goldacre R, Gair S, Curnow P, Bray $\mathrm{M}$, et al. COVID-19 pandemic and admission rates for and management of acute coronary syndromes in England. Lancet. 2020;396:381-9.

12. Papafaklis MI, Katsouras CS, Tsigkas G, Toutouzas K, Davlouros $\mathrm{P}$, Hahalis GN, et al. "Missing" acute coronary syndrome hospitalizations during COVID-29 era in Greece: medical care avoidance combined with a true reduction in incidence? Clin Cardiol. 2020;43:1142-9.

13. Kudo N, Tanaka A, Ishii H, Uemura Y, Takagi K, Iwama M, et al. Prevalence of acute coronary syndrome during the pandemic of COVID-19 in the Tokai region of Japan. Nagoya J Med Sci (in press)

14. Yasuda $\mathrm{Y}$, Ishiguchi H, Ishikura M, Yoshida M, Imoto K, Sonoyama K, et al. Incidence and demographic trends for acute coronary syndrome in a non-epidemic area during the coronavirus disease pandemic in Japan - a 2-institutional observational study. Circ Rep. 2021;3:95-9.

15. Kitahara S, Fujino M, Honda S, Asaumi Y, Kataoka Y, Otsuka F, et al. COVID-19 pandemic is associated with mechanical complications in patients with ST-elevation myocardial infarction. Open Heart. 2021;8:e01497.

16. Ueda Y, Tahara Y, Itoh T, Tsujita K, Sakuma M, Amano T, Sato K, Taniguchi T, Nishiyama C, Konno S, Iwami T, Murohara T, Node K. New strategy to prevent acute myocardial infarction by public education - a position statement of the committee on public education about emergency medical care of the Japanese Circulation Society. Circ J. 2021;85:319-22.

17. Japanese Ministry of Health, Labour and Welfare. https://www. mhlw.go.jp/toukei/saikin/hw/jinkou/geppo/s2020/12.html. Accessed 20 Mar 2021

18. Kimura K, Kimura T, Ishihara M, Nakagawa Y, Nakao K, Miyauchi K, et al. JCS 2018 guideline on diagnosis and treatment of acute coronary syndrome. Circ J. 2019;83:1085-196.

Publisher's Note Springer Nature remains neutral with regard to jurisdictional claims in published maps and institutional affiliations. 